\title{
Hue detectability thresholds as a function of chronological age'
}

\begin{abstract}
Abstraet
One hundred eighty normal children aged 7 to 12 years exhibited no ontogenetic trends in their hue detection thresholds, nor were these thresholds correlated with $I Q$. Of the three hues studied, orange yielded significantly lower thresholds than either green or blue which did not differ from each other. These data contrast with those obtained for contour detection thresholds which rose as a function of increasing chronological age. It was suggested that geometrical illusion figures based upon hue contrast alone, rather than brightness contrast, would not produce the usual ontogenetic trends.

\section{Imitrodienetion}

A recent study by the present investigator (Pollack, 1963) has demonstrated that contour detectability as a function of light intensity declines with chronological age through childhood. Evidence quoted by Weale (1961a, 1961b) and Hinchcliffe (1962) indicates a loss of peripheral visual sensitivity with increasing age due to such factors as increasing lenticular density, lenticular pigmentation, decreasing pupil size, and perhaps increasing retinal pigmentation. Brody (1955) found a correlation between chronological ages ranging from 0 to 95 years and cerebral cortex cell counts of primary projection areas of -.99 , which may indicate central loss of sensitivity in addition to peripheral losses. The increase in contour detectability thresholds may be accounted for by these physiological processes without appeal to any experiential or intellectual factors.

If such is indeed the case, then it might be expected that thresholds for the detection of various hues should also rise through the same range of increasing ages that Pollack (1963) previously investigated. The present study was designed to test this hypothesis with three hues, an orange, a green, and a blue.

In the earlier work it was found thatalthough contour detection thresholds rose with chronological age and therefore with mental age, there was a significant $(p<.05)$ negative correlation between IQ and threshold. A study to determine the correlation between hue detectability thresholds and IQ was planned in order to test generality of the earlier findings. No hypothesis was advanced concerning possible differential developmental sequences as a function of hue differences, but the use of three hues in the investigation made possible an evaluation of this dimension as well.

\section{Method}

Subjects Ss were 180 children, 7-12 years old. All were pupils in a suburban elementary school. ${ }^{2}$ They were divided into six age groups, $(7,8,9,10,11$, and 12 years old) of $30 \mathrm{Ss}$ each. Each age group was in turn divided at random into three groups of $10 \mathrm{Ss}$, each group to experience only one hue. Children were tested using a Cohen chart and the American Optical Company's Pseudoisochromatic Plates, and only children having 20/30 uncorrected vision or better and no color defects were used.

Apparatus The apparatus consisted of a Gerbrands variable speed, variable sector color mixer and a Munsell variable speed color mixer. Four Munsell color disks were employed: orange, $5 \mathrm{Y}-\mathrm{R} 5 / 8$; green, 5 G 5/8; blue, 5 P-B 5/8; and gray, N 5 Y. As can be seen, the disks were equated for lightness (Munsell value 5) and the hues also were equated for chroma or saturation (Munsell value 8). A colored disk and a gray one were fixed to the Gerbrands apparatus to provide the variable stimulus while a gray disk fixed to the Munsell apparatus served as the standard.

The color wheels were housed in a viewing device constructed for this experimentby the Gaertner Scientific Corporation, described fully elsewhere (Pollack \& Magerl, 1965). The device was a light-tight Masonite box painted black on the inside. It permitted binocular viewing of two round apertures, each of which subtended a visual angle of $1^{\circ}$. The standard color wheel containing the gray disk was placed on the left at an angle of $90^{\circ}$ to the line of sight. It was illuminated by illuminant " $\mathrm{C}$ " (color temperature $6500^{\circ} \mathrm{K}$ ) and reflected through the left aperture to the eyes by means of a mirror set at an angle of $45^{\circ}$ to the line of sight. The variable color wheel was placed on the right, also $90^{\circ}$ to the line of sight. It too was illuminated by illuminant " $\mathrm{C}$ " and reflected through the right apeture to the eyes by means of a mirror set at an angle of $45^{\circ}$ to the line of sight. A shutter in front of the right, or variable, color aperture permitted a fixed exposure time for each color setting.

Procedure In each group, $\mathrm{S}$ was shown a hue-plusgray combination on the variable mixer and gray alone on the standard mixer, both mixers operating at a speed beyond fusion. First, $\mathrm{S}$ was shown a mixture in which the amount of gray was so small that the variable was clearly different from the standard. Then the variable was shown with the amount of gray so large that no difference could be detected. It was stated that sometimes the variable would appear different from the standard and that $S$ should respond with the word "Yes" if they were the same and "No" if they 


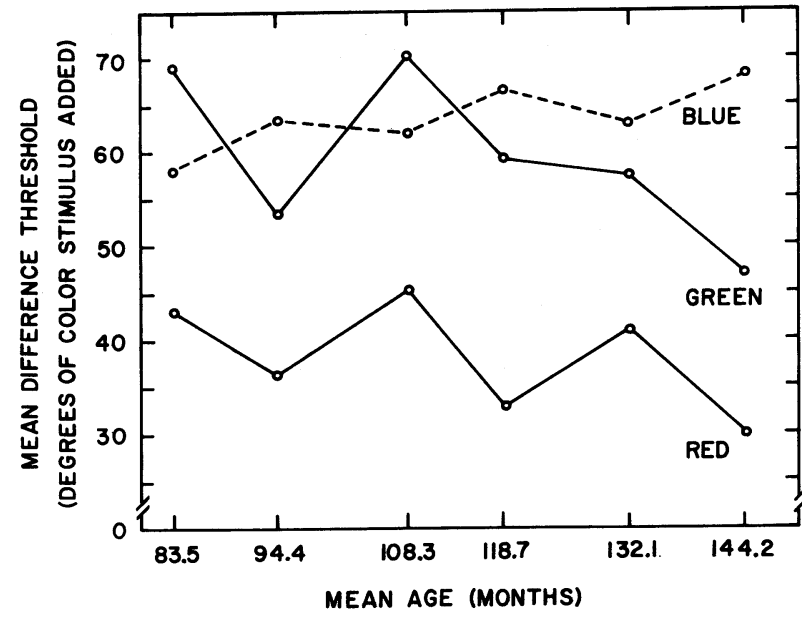

Fig 1. Hue detection threshold as a function of chronological age. Threshold measures are given in terms of mean degrees of hue added to gray on the variable color wheel

were not. The method of limits was used-six ascending and six descending trials in counterbalanced order. Color mixtures were altered in steps of $5^{\circ}$. Each setting was exposed for $500 \mathrm{msec}$. Between settings the standard gray stimulus was visible, but the closed shutter in front of the variable prevented $\mathrm{S}$ from seeing the color settings being changed. A rest period of 30 sec. was given at the end of each trial, and an additional $2 \mathrm{~min}$. rest period was inserted half-way through the series of trials.

\section{Results and Diseussion}

Thresholds were the calculated $50 \%$ points for each $S$ based on the 12 trials. These data were analyzed by means of a double classification analysis of variance design ( 3 colors by 6 age groups) with the within-cell variance as the error term. The analysis yielded a main effect of hue ( $F=43.54$, df 2 and 162) but no effect of age ( $F=1.15$, df 5 and 162) and no significant interaction between age and hue ( $F=1.47$, df 10 and 162). Comparing pairs of hue thresholds by means of t-tests showed that those for blue and green did not differ from each other, but the thresholds for both were significantly higher than that for orange (see Fig. 1). No explanation is offered for the hue differences. If there are age changes in hue detectability, then they occur at ages younger than 7 or older than 12 .

Since the analysis of variance showed no age effect and no age-hue interaction, product moment correlations were calculated between threshold scores for each color and IQ scores (Finch, 1957) with all age groups combined. The correlation with orange threshold was -.16 $(\mathrm{N}=32)$, with green -.31 ( $=31)$, and with blue .335 $(\mathrm{N}=29)$, none of which was statistically significant。

Comparing these results with those obtained on contour detectability (Pollack, 1963), it appears clear that the two processes of contour detection and hue detection are underlain by different receptor systems with different ontogenetic courses. Furthermore, while S's sensitivity to contour showed minimal significant correlation with $\mathrm{IQ}$, sensitivity to hue showed no such correlation. One would expect, upon the basis of the results obtained here, that a Type I (Pollack, 1963) illusion involving a figure contrasting with its ground only in hue would not show the commonly-seen ontogenetic course of decreasing magnitude with increasing age between ages 7 and 12 .

\section{References}

Brody, H Organization of cer: ral cortex study of aging in human cerebral cortex J. comp. Neurol., 1955, 102, 511-556

Finch. F. H. Kuhlman-Finch Tests. Minneapolis, Minn.: American Guidance Service, Inc , 1957

Pollack. R H Contour detectability threshold as a function of chronological age Percept. mot. Skills, 1963, 17, 411-417

Pollack, R. H., \& Magerl G E A binocular viewing apparatus for sensory threshold studies in children Percept. mot. Skills, $1965,20,127-130$

Weale. $\mathbf{R} \boldsymbol{A}$ Notes on the photometric significance of the human crystalline lens. Vis. Res., 1951a, 1, 183-191

Weale, R. A. Retinal illumination and age. Trans. Illum. Eng. Soc., London, 1961b, 26, 95-100.

\section{Notes}

1 This research was supported in part by Research Grant No HD 01433-01, awarded by the National Institute of Child Health and Human Development

2 The author wishes to acknowledge the assistance and cooperation of Mr. Richard W Clifford, Principal of the Rugen School, Glenview, Illinois and his staff and of John H Spriger, Ph D., superintendent of Illinois School District 34 Acknowledgement is also extended to Dorothy Nelson, $\mathrm{Ph} \mathrm{D}$, of the Institute for Juvenile Research who collected the data with no knowledge of the author's expectations or biases 